Overview of the RFX fusion science program

This article has been downloaded from IOPscience. Please scroll down to see the full text article.

2011 Nucl. Fusion 51094023

(http://iopscience.iop.org/0029-5515/51/9/094023)

View the table of contents for this issue, or go to the journal homepage for more

Download details:

IP Address: 150.178.3.9

The article was downloaded on 19/01/2012 at $14: 20$

Please note that terms and conditions apply. 


\section{Overview of the RFX fusion science program}

P. Martin $^{1}$, J. Adamek ${ }^{2}$, P. Agostinetti ${ }^{1}$, M. Agostini ${ }^{1}$, A. Alfier ${ }^{1}$, C. Angioni ${ }^{3}$, V. Antoni ${ }^{1}$, L. Apolloni ${ }^{1}$, F. Auriemma ${ }^{1}$, O. Barana ${ }^{1}$, S. Barison ${ }^{4}$, M. Baruzzo ${ }^{1}$, P. Bettini ${ }^{1}$, M. Boldrin ${ }^{1}$, T. Bolzonella ${ }^{1}$, D. Bonfiglio $^{1}$, F. Bonomo ${ }^{1}$, A.H. Boozer ${ }^{5,6}$, M. Brombin ${ }^{1}$, J. Brotankova ${ }^{2}$, A. Buffa ${ }^{1}$, A. Canton ${ }^{1}$, S. Cappello ${ }^{1}$, L. Carraro ${ }^{1}$, R. Cavazzana ${ }^{1}$, M. Cavinato ${ }^{1,7}$, L. Chacon ${ }^{8}$, G. Chitarin ${ }^{1}$, W.A. Cooper ${ }^{9}$, S. Dal Bello ${ }^{1}$, M. Dalla Palma ${ }^{1}$, R. Delogu ${ }^{1}$, A. De Lorenzi ${ }^{1}$, G. De Masi ${ }^{1}$, J.Q. Dong ${ }^{10}$, M. Drevlak ${ }^{11}$, D.F. Escande ${ }^{12}$, F. Fantini ${ }^{1}$, A. Fassina ${ }^{1}$, F. Fellin ${ }^{1}$, A. Ferro ${ }^{1}$, S. Fiameni ${ }^{4}$, A. Fiorentin ${ }^{1}$, P. Franz ${ }^{1}$, E. Gaio ${ }^{1}$, X. Garbet ${ }^{13}$, E. Gazza ${ }^{1}$, L. Giudicotti ${ }^{1}$, F. Gnesotto ${ }^{1}$, M. Gobbin ${ }^{1}$, L. Grando ${ }^{1}$, S.C. Guo ${ }^{1}$, Y. Hirano ${ }^{14}$, S.P. Hirshman ${ }^{7}$, S. Ide $^{15}$, V. Igochine $^{3}$, Y. In ${ }^{16}$, P. Innocente ${ }^{1}$, S. Kiyama ${ }^{14}$, S.F. Liu ${ }^{17}$, Y.Q. Liu ${ }^{18}$, D. Lòpez Bruna ${ }^{19}$, R. Lorenzini ${ }^{1}$, A. Luchetta ${ }^{1}$, G. Manduchi ${ }^{1}$, D.K. Mansfield ${ }^{6}$, G. Marchiori ${ }^{1}$, D. Marcuzzi ${ }^{1}$, L. Marrelli ${ }^{1}$, S. Martini ${ }^{1}$, G. Matsunaga ${ }^{15}$, E. Martines ${ }^{1}$, G. Mazzitelli $^{20}$, K. McCollam ${ }^{21}$, S. Menmuir ${ }^{1}$, F. Milani ${ }^{1}$, B. Momo ${ }^{1}$, M. Moresco ${ }^{1}$, S. Munaretto ${ }^{1}$, L. Novello ${ }^{1}$, M. Okabayashi ${ }^{6}$, S. Ortolani ${ }^{1}$, R. Paccagnella ${ }^{1}$, R. Pasqualotto ${ }^{1}$, M. Pavei ${ }^{1}$, G.V. Perverezev ${ }^{3}$, S. Peruzzo ${ }^{1}$, R. Piovan $^{1}$, P. Piovesan $^{1}$, L. Piron $^{1}$, A. Pizzimenti ${ }^{1}$, N. Pomaro ${ }^{1}$, N. Pomphrey ${ }^{6}$, I. Predebon ${ }^{1}$, M.E. Puiatti ${ }^{1}$, V. Rigato $^{1}$, A. Rizzolo $^{1}$, G. Rostagni ${ }^{1}$, G. Rubinacci $^{22}$, A. Ruzzon ${ }^{1}$, H. Sakakita ${ }^{14}$, R. Sanchez ${ }^{8,23}$, J.S. Sarff ${ }^{21}$, F. Sattin ${ }^{1}$, A. Scaggion ${ }^{1}$, P. Scarin ${ }^{1}$, W. Schneider ${ }^{11}$, G. Serianni ${ }^{1}$, P. Sonato ${ }^{1}$, E. Spada ${ }^{1}$, A. Soppelsa ${ }^{1}$, S. Spagnolo ${ }^{1}$, M. Spolaore ${ }^{1}$, D.A. Spong ${ }^{8}$, G. Spizzo ${ }^{1}$, M. Takechi ${ }^{15}$, C. Taliercio ${ }^{1}$, D. Terranova ${ }^{1}$, V. Toigo ${ }^{1}$, M. Valisa ${ }^{1}$, M. Veranda ${ }^{1}$, N. Vianello ${ }^{1}$, F. Villone $^{24}$, Z. Wang ${ }^{1}$, R.B. White ${ }^{6}$, D. Yadikin $^{3}$, P. Zaccaria ${ }^{1}$, A. Zamengo ${ }^{1}$, P. Zanca ${ }^{1}$, B. Zaniol ${ }^{1}$, L. Zanotto ${ }^{1}$, E. Zilli ${ }^{1}$, G. Zollino ${ }^{1}$ and M. Zuin ${ }^{1}$

\footnotetext{
${ }^{1}$ Consorzio RFX, Associazione EURATOM-ENEA sulla Fusione, 35137 Padova, Italy 2 Institute of Plasma Physics, Association EURATOM-IPP.CR, Prague, Czech Republic ${ }^{3}$ Max-Planck-Institut für Plasmaphysik, EURATOM Association, 85748 Garching, Germany ${ }^{4}$ CNR-IENI, Corso Stati Uniti 4, 35127 Padova, Italy

${ }^{5}$ Department of Applied Physics and Applied Mathematics, Columbia University, New York, NY, USA

${ }^{6}$ Princeton Plasma Physics Laboratory, Princeton, NJ, USA

${ }^{7}$ Fusion for Energy Joint Undertaking, 08019 Barcelona, Spain (present address)

${ }^{8}$ ORNL Fusion Energy Division, Oak Ridge, TN, USA

${ }^{9}$ EPFL, Association EURATOM-Confederation Suisse, Centre de Recherches en Physique des Plasmas, Lausanne, Switzerland

${ }^{10}$ Institute for Fusion Theory and Simulation, Zhejiang University, Hangzhou, Southwestern Institute of Physics, Chengdu, People's Republic of China

${ }^{11}$ Max-Planck-Institut für Plasmaphysik, EURATOM Association, Greifswald, Germany

12 UMR 6633 CNRS-Université de Provence, Marseille, France

13 CEA, IRFM, F-13108 Saint Paul Lez Durance, France

${ }^{14}$ Plasma Frontier Group, Energy Technology Research Institute, National Institute of Advanced Industrial Science and Technology (AIST), Tsukuba Central 2, 1-1-1 Umezono, Tsukuba, Ibaraki 305-8568, Japan

15 Japan Atomic Energy Agency, Naka, Ibaraki 311-0193, Japan

${ }^{16}$ FAR-TECH, Inc., 3550 General Atomics Ct, San Diego, CA 92121, USA
} 
${ }^{17}$ Department of Physics, Nankai University, Tianjin 300071, People's Republic of China

${ }^{18}$ EURATOM/CCFE Fusion Association, Culham Science Centre, Abingdon, Oxfordshire OX14 3DB, UK

${ }^{19}$ Laboratorio Nacional de Fusión, Asociación EURATOM-CIEMAT, Madrid, Spain

${ }^{20}$ Centro Ricerche Energia ENEA Frascati, Associazione Euratom-ENEA sulla Fusione, Frascati, Italy

${ }^{21}$ Department of Physics, University of Wisconsin, Madison, WI, USA

${ }^{22}$ Ass. Euratom/ENEA/CREATE, DIEL, Università di Napoli Federico II, Napoli, Italy

${ }^{23}$ Universidad Carlos III de Madrid, Madrid, Spain

${ }^{24}$ Ass. Euratom/ENEA/CREATE, DAEIMI, Università di Cassino, Cassino, Italy

E-mail: piero.martin@igi.cnr.it

Received 15 April 2011, accepted for publication 14 June 2011

Published 31 August 2011

Online at stacks.iop.org/NF/51/094023

\begin{abstract}
This paper summarizes the main achievements of the RFX fusion science program in the period between the 2008 and 2010 IAEA Fusion Energy Conferences. RFX-mod is the largest reversed field pinch in the world, equipped with a system of 192 coils for active control of MHD stability. The discovery and understanding of helical states with electron internal transport barriers and core electron temperature $>1.5 \mathrm{keV}$ significantly advances the perspectives of the configuration. Optimized experiments with plasma current up to $1.8 \mathrm{MA}$ have been realized, confirming positive scaling. The first evidence of edge transport barriers is presented. Progress has been made also in the control of firstwall properties and of density profiles, with initial first-wall lithization experiments. Micro-turbulence mechanisms such as ion temperature gradient and micro-tearing are discussed in the framework of understanding gradient-driven transport in low magnetic chaos helical regimes. Both tearing mode and resistive wall mode active control have been optimized and experimental data have been used to benchmark numerical codes. The RFX programme also provides important results for the fusion community and in particular for tokamaks and stellarators on feedback control of MHD stability and on three-dimensional physics. On the latter topic, the result of the application of stellarator codes to describe three-dimensional reversed field pinch physics will be presented.
\end{abstract}

(Some figures in this article are in colour only in the electronic version)

\section{Executive summary: RFX mission, highlights and open questions}

This first section is intended to serve as an executive summary of the paper: it summarizes the mission of RFX-mod and its connection with the European and international fusion program, the major highlights of the RFX fusion science program obtained in the period between the 2008 and 2010 IAEA Fusion Energy Conferences, the open questions and perspectives of RFP physics.

The rest of the paper is organized as follows: in section 2 some basic information on the RFP configuration is provided. Section 3 describes the activity for the setting up of highcurrent scenarios in RFX-mod. Internal transport barriers (ITBs) and helical states are the subject of section 4. Section 5 is devoted to edge transport barriers, while section 6 describes the progress and the achievements on three-dimensional (3D) physics. Real-time feedback control of MHD stability is the subject of sections 7 and 8, while conclusions are drawn in section 9.

\subsection{Mission}

The mission-oriented RFX fusion science program is adapting to the new challenges of the fusion community and it is shaped to provide a focused contribution to ITER and its accompanying program.
RFX-mod is a flexible reversed field pinch (RFP) toroidal device (major radius $R=2 \mathrm{~m}$ and minor radius $a=0.46 \mathrm{~m}$ ) with plasma current $2 \mathrm{MA}$ and volume $10 \mathrm{~m}^{3}[1]$. As in all RFPs, plasma heating is purely ohmic; RFP could in principle obtain fusion power with ohmic heating only, and with magnetic field much smaller than in a tokamak-avoiding superconducting coils. RFX-mod is equipped with a very powerful system of active coils for feedback control of plasma MHD stability: 192 coils, independently driven, cover the whole plasma surface.

The major challenges of RFP research, and of RFX-mod in particular, are (a) rapidly advancing its performance, to assess the viability of the RFP approach to fusion; (b) providing a state-of-the-art contribution to the global task of feedback control of MHD stability, with experiments done both in RFP and in tokamak configuration; (c) focusing on the key topic of $3 \mathrm{D}$ magnetic shaping in a growing collaboration with the stellarator community; (d) training a new generation of fusion scientists.

\subsection{Highlights}

Since the last Fusion Energy Conference (FEC) in 2008 [2] the RFX-mod fusion science program has crossed several important milestones.

After extensive optimization of 1.5 MA regimes-where the improved performance of the self-organized spontaneous 


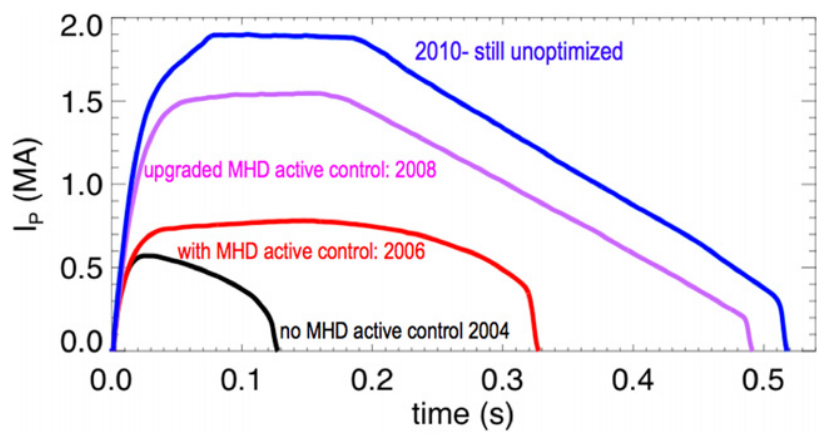

Figure 1. Plasma current waveforms obtained in RFX-mod since its restart in December 2004.

helical equilibrium with a single helical axis (SHAx) has been broadly documented [3] - the program focused on the exploration of plasma currents towards the maximum RFX-mod design value of $2 \mathrm{MA}$. A number of technical improvements (including active cooling of the primary windings [4]) and the development of a new start-up scenario [5]) allowed for physics exploration at a plasma current $I_{\mathrm{p}} \approx$ 1.8 MA and for preliminary experiments at $2 \mathrm{MA}$. The latter, though far from being optimized, show no technical limit for operation at $2 \mathrm{MA}$ and confirm the robustness of the device. Examples are shown in figure 1.

Significant progress on the understanding of the SHAx state has been made. SHAx states have strong electron internal transport barriers (eITBs) [6], where a minimum electron heat diffusivity $\chi_{\mathrm{e}, \min } \approx 5 \mathrm{~m}^{2} \mathrm{~s}^{-1}$ is measured. This coincides with a region of null magnetic shear, where the density of rational surfaces and thus the probability of mode coupling are lowered. This is similar to what is described in [7] for tokamaks, where in the presence of dominant electron heating a reversed magnetic shear and especially a shear null represent a sufficient condition for a strong electron barrier to develop [8,9]. In RFX-mod, the SHAx states are accompanied by significant $\boldsymbol{E} \times \boldsymbol{B}$ sheared poloidal flow. Although the role of flow shear in the formation of the ITBs is not yet fully explained, the presence of a sheared poloidal flow is indeed expected by MHD codes and the existence of a flow pattern modulated by the helical deformation is experimental evidence [10]. The value of $\chi_{e \text {,min }}$ expressed in Bohm units is consistent with data from similar tokamak and stellarator databases, confirming that in the barrier region RFP transport approaches the quality of other configurations. The experimental particle transport coefficient in the helical core is reduced compared with the multiple helicity $(\mathrm{MH})$ case, reaching values of the same order of magnitude of the volume-averaged diffusivity estimated with a test particle approach in helical geometry. The $1 / v$ transport regime (super-banana effects), typical of an unoptimized stellarator, is not found in numerical simulation [11]. Strong edge electron transport barriers are also observed, though not necessarily linked with single helicity $(\mathrm{SH})$ states. They lead to electron pressure pedestal, with $\nabla T_{\mathrm{e}} \approx 80 \mathrm{keV} \mathrm{m}^{-1}$. A suite of numerical codes has been assembled to study helical states, in a strong partnership with the stellarator community [11, 12]. In particular, a significant effort is being made to adapt stellarator numerical tools to RFP. This has the advantage of improving the understanding of RFP 3D features and at the same time of providing an effective possibility to benchmark stellarator codes against experimental data and to improve the 3D fusion physics knowledge basis.

Helical eITBs appear at moderate density (Greenwald fraction $n / n_{\mathrm{g}} \leqslant 0.25$ ), consistent with the evidence that the magnetic bifurcation leading to SHAx states occurs at a low collisionality. Quasi-single helicity (QSH) states are obtained up to $n / n_{\mathrm{g}} \approx 0.35$, but without eITBs. Such a density limit is attributed to localized edge density accumulation and plasma cooling, in combination with helical plasma-wall interaction (PWI) $[13,14]$.

A rich program on active control of MHD stability has provided a large number of new results [15], in collaboration with the tokamak community. Real-time control experiments have been performed operating RFX-mod both as RFP and as tokamak, to address control of resistive wall modes (RWMs), tearing modes (TMs) and magnetic field errors. Improvements are obtained by including in control models toroidal geometry and non-uniformity of the passive structures, and by taking into account the coupling between sensor and coils in MIMO models. Mode tracking experiments have been successfully used to apply a non-zero SH reference edge magnetic field, to sustain a single helical mode at the desired edge amplitude. RWM non-rigidity was studied. Downgrading and reconfiguring the 192 active coils showed that the most unstable RWM can still be controlled by reducing the active coil coverage to $25 \%$ of the plasma surface. This was performed in collaboration with JT-60SA to help the design of its set of active coils. A new integrated 'flightsimulator' for closed loop control experiments was developed and benchmarked.

RFX-mod can be run as a tokamak, with $120 \mathrm{kA}$ plasma current lasting up to $1.2 \mathrm{~s}$. In this configuration a currentdriven $(2,1)$ RWM is observed in ramped current plasmas as $q_{\text {edge }} \approx 2$. This mode is actively stabilized so that for the first time a feedback stabilized $q_{\text {edge }} \approx 2$ tokamak plasma is run without disruptions.

\subsection{Challenges and perspectives}

The results obtained since the 2008 FEC proved the transformational nature of the helical RFP state. RFX-mod routinely operates at a high current in regimes of very low magnetic transport in the core, as a result of spontaneous self-organization. Two main open issues now represent the remaining challenges on confinement.

The first one concerns density control: at a high current it is difficult to achieve helical states with a high density. In general, high first-wall recycling prevents a high-current, high-density operation. This leads to an edge-peaked density profile and edge cooling, which makes the edge plasma highly resistive. This is a particularly critical issue since, if the edge is resistive, more input power is required to drive the poloidal current necessary to sustain the RFP configuration. Inherently associated with this is the second open issue, which deals with the ohmic input power that is still higher than expected, with a negative impact on the global energy confinement time. One reason for this is the edge density, as just explained, but other causes might be present, linked for example with a still unoptimized magnetic boundary. To improve density control, hydrogen pellet fuelling is combined 
with wall-recycling control by means of lithization [13]. The first tests with lithium give better density control and more peaked profiles. A significant core density increase is obtained with $\mathrm{H}$ pellets.

In the absence of 'first-principles-based' showstopper, most of the limits appear to be operational (e.g. first-wall or active control optimization), and therefore solvable. The next steps in the RFX science program will address these challenges relying on both a clear path and robust and flexible tools available to follow it. The path to exploitation of $2 \mathrm{MA}$ RFX-mod with improved first-wall conditioning and optimized magnetic boundary, supported by a growing effort on 3D modelling, is open and worth being followed. In addition to that, starting from the experiments described in this paper, optimization of real-time feedback control will proceed, in particular for those topics more relevant for application to tokamaks.

\section{The RFP magnetic configuration}

The RFP is a toroidal configuration for the magnetic confinement of thermonuclear plasmas. The RFP shares with the tokamak similar features, for example the presence of two components of the confining magnetic field: a toroidal component $B_{\mathrm{t}}$ and a poloidal one, $B_{\mathrm{p}}$, and the pinch effect. The latter means that a toroidal electric current is driven in a plasma, which is embedded in a toroidal magnetic field. Different from the tokamak, the applied toroidal magnetic field is extremely small. The plasma toroidal magnetic field, which is of the same order of magnitude as the poloidal field - another significant difference with respect to the tokamak where the toroidal magnetic field is one order of magnitude larger than the poloidal-is mainly produced by electrical currents flowing in the plasma through a self-organization process and changes sign at the plasma edge with respect to the core.

Given its magnetic topology, the RFP exploits the advantage of an easier technology involved in the electromagnetic system. This might be the case even for a reactor, where there might be no need for large superconducting magnetic coils; there is freedom in the choice of the aspect ratio and in principle ignition should be achievable with ohmic heating only.

A price to be paid for exploiting these advantages concerns magnetohydrodynamic (MHD) stability. The safety factor $q$ is in fact lower than 1 across the whole plasma, and negative at the edge. Figure $2(a)$ shows the $q$ profiles of an RFP compared with those of tokamaks and stellarators. In the RFP $q$ profile there are multiple resonant surfaces in the plasma, in particular for modes with poloidal mode numbers $m=0$ and 1 . This is shown in figure $2(b)$, which reports a typical RFP $q$ profile. Two main kinds of global, current-driven MHD instabilities may be present in a RFP with resistive wall: (i) resistive kink/TMs, which are resonant in the plasma and are intrinsically linked to the sustainment of the configuration through the aforementioned self-organization process. (ii) RWMs, which are non-resonant ideal modes, slowed down by the resistive wall. RWMs in RFP are current driven and are present also at low plasma beta.

TM spectrum detailed structure depends on the plasma regime $[2,16,17]$. In the $\mathrm{MH}$ regime the spatial spectrum
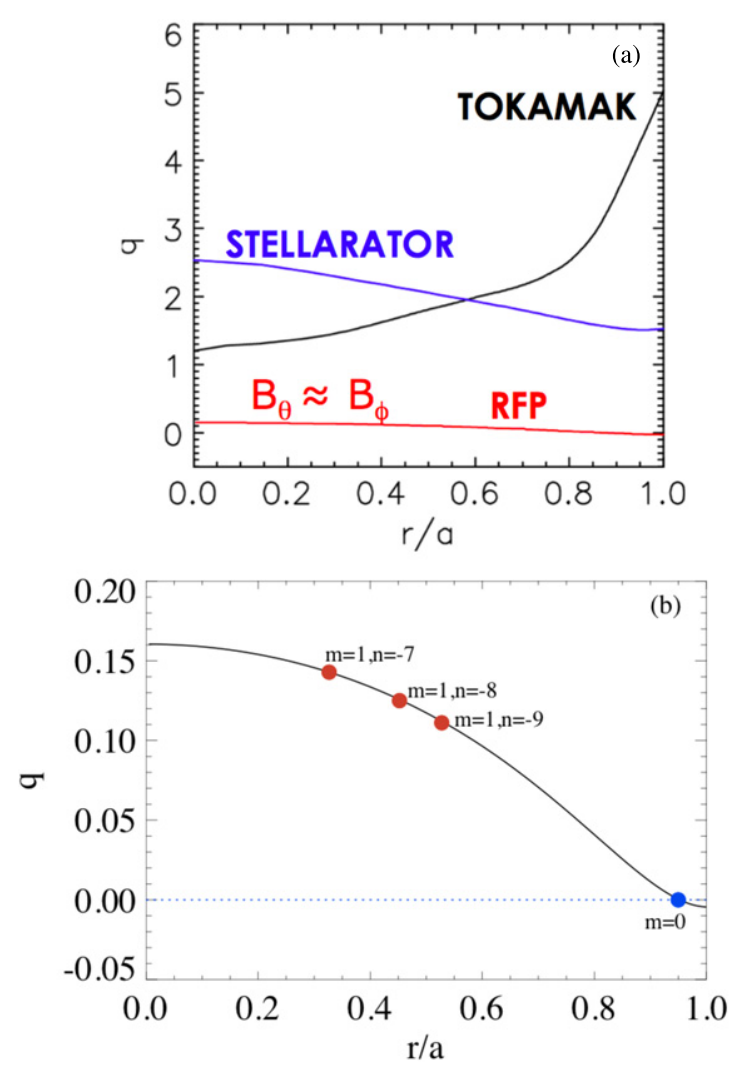

Figure 2. (a) Typical RFP, tokamak and stellarator safety factor profiles. (b) Typical RFP safety factor profile (RFX-mod geometry used here).

of $m=1$ modes is rather rich and several modes are simultaneously present in the plasma with a significant amplitude. This is not positive for confinement. In the QSH regime the spectrum is almost monochromatic: one $m=1$ mode with a well-defined toroidal mode number $n$ dominates over the other helicities, dubbed secondary modes. Recent results from RFX-mod show that, when the plasma current is raised above $\approx 1.5 \mathrm{MA}$, the $\mathrm{QSH}$ regime features the spontaneous occurrence of a self-organized helical equilibrium with a SHAx, reduced magnetic fluctuations and strong eITB [3]. The SHAx equilibrium has a helical core with $m=1$, $n=7$ symmetry embedded in an almost axis-symmetric boundary. Core electron temperature reaches $1.7 \mathrm{keV}$.

The traditional way to achieve RFP stability exploited the presence of a thick conducting wall. During the last years the RFP community has developed extensive projects on active control of MHD stability, and in particular on feedback control in devices with a thin wall. Two RFP devices, RFX-mod and EXTRAP T2R [18], have been equipped with a resistive wall and with coil systems for active control of plasma stability, which are the most advanced ever realized in a fusion device. The exploitation of these feedback systems is leading to very significant improvements in the RFP performance [19]—for example favouring the presence of helical states. A smoother magnetic boundary is provided by feedback control. This reduces edge TM amplitude, and provides for a milder PWI. Feedback allows the RFP to give key contributions to the science and technology of real-time active control of MHD stability in fusion devices [20]. 

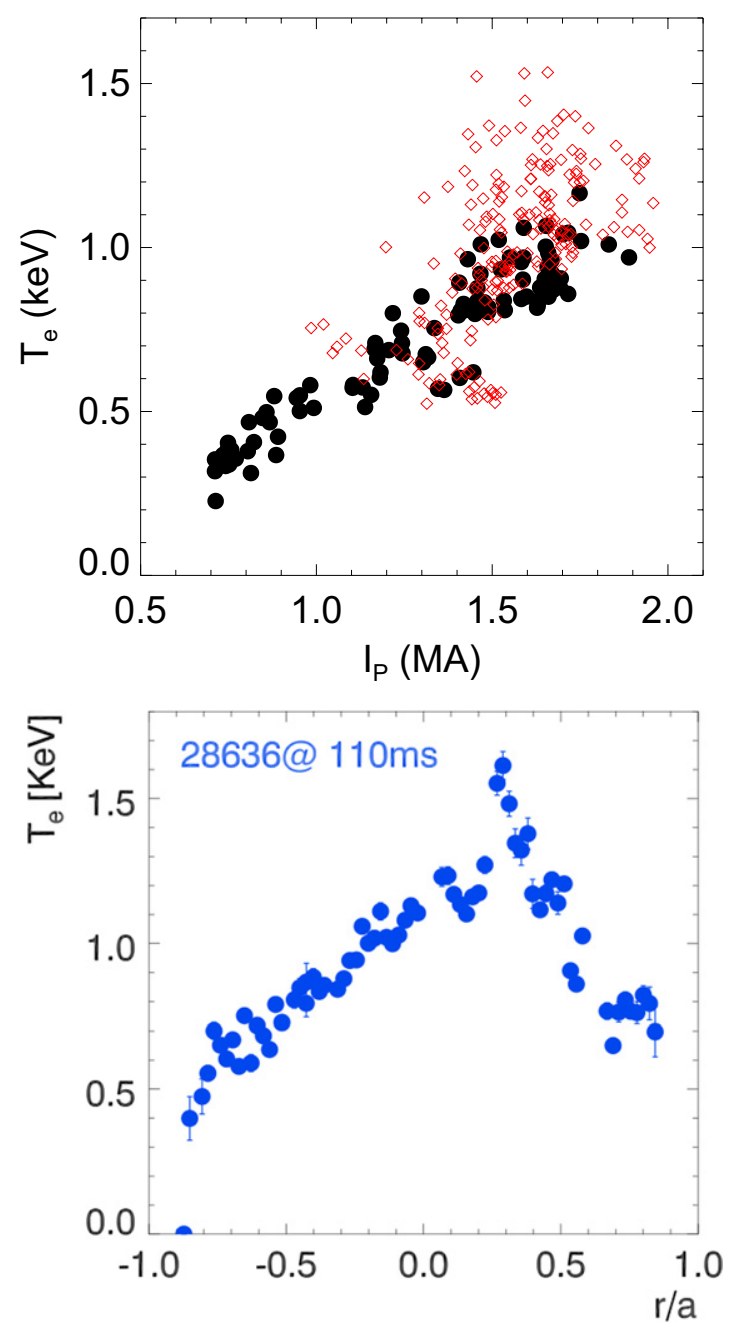

Figure 3. Top: core $T_{\mathrm{e}}$ versus $I_{\mathrm{p}}$ for two different ranges of $n / n_{\mathrm{gw}}$ : $[0.05,0.13]$ (red diamonds) and [0.14,0.21] (black circles). Each point is an average during $I_{\mathrm{p}}$ flattop. Bottom: typical high-current electron temperature profile.

\section{Optimization of high-current operation}

RFX-mod is in a unique position to test scaling of plasma performance with current and to explore RFP physics in the MA range. Plasma current is a major control parameter for performance. Past experience, reported in the previous FEC [2], indicated significant changes in RFX-mod plasma performance as plasma current was raised above $1 \mathrm{MA}$. The central electron temperature increase with $I_{\mathrm{p}}$ up to values $\geqslant 1.5 \mathrm{keV}$ (see figure 3, which shows the behaviour of the core electron temperature versus plasma current and a typical electron temperature profile at high plasma currents) is confirmed by recent experiments up to $I_{\mathrm{p}} \approx 1.8 \mathrm{MA}$. Electron heating translates in increased magnetic Lundquist number $S$. The decrease in the amplitude of internally resonant TMs with increasing $S$ was previously observed in MST [21] and confirmed by RFX-mod in a broader $S$ range [2]. The novelty from RFX-mod is that the positive $S$-scaling of high $n$ mode amplitudes (called secondary modes) is synergic with the dependence on $S$ and plasma current of the innermost resonant mode amplitude ( $m=1, n=-7$, the dominant mode). This is the background for the emergence of the helical SHAx states

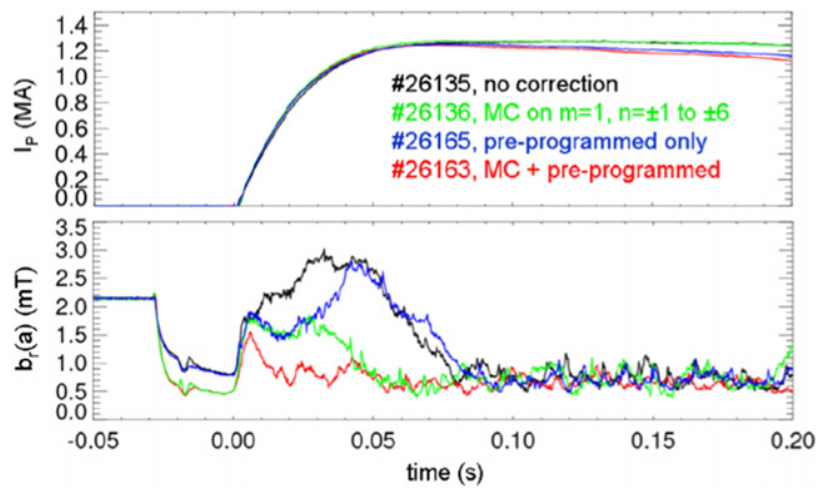

Figure 4. Current waveforms (top) and different error field control schemes (bottom). Mode control with feed-forward error control provides the best correction (red curve).

at $I_{\mathrm{p}} \geqslant 1.5 \mathrm{MA}[2,3]$. The growth of the dominant mode leads to core ordered helical topology, with a SHAx. The decrease in secondary modes drives magnetic chaos reduction.

Producing high-quality plasmas at high currents, close to the machine design limit, calls for careful scenario design, which will be described in the following subsections.

\subsection{High-current discharge set-up}

Core elements of RFX-mod power supply are modular ac/dc converters, which feed the machine windings. A total of 12 converters were equally divided into three groups of 4 each, for the toroidal coil, and for the magnetizing windings (4 to build the primary current which is used to set up the configuration and raise the current, and 4 for the application of the toroidal loop voltage, which sustains the plasma current during the flattop phase).

The 12 modular ac/dc converters that feed the toroidal and poloidal field circuits have recently been reconfigured to make more energy available for the magnetizing windings that drive the loop voltage, at the expense of the bias toroidal field. Since currents flowing in the plasma produce most of the toroidal field in an RFP, only a small amount needs to be produced with external coils. More energy is available to extend the initial current ramp-up and easily reach $2 \mathrm{MA}$ and beyond [5]. An example of this kind of set-up is shown in figure 1 for the highest $I_{\mathrm{p}}$ discharge (blue curve, label '2010').

\subsection{Error field correction}

In RFX-mod, during the current ramp-up phase, the vertical magnetic field penetrates faster through the two poloidal gaps in the shell and reproducible field errors of a few $\mathrm{mT}$ are observed, toroidally localized at the gap positions. Reference signals recorded in dry runs are then used for active correction in the feed-forward mode. Reference signals are computed with a dynamic decoupler [22] (described in section 7), which uses the frequency dependence of the coupling between actuators and sensors. Adding to the pre-programmed error control the feedback for the main modes contributing to the error field (i.e. $m=1, n= \pm 2, n= \pm 4, n= \pm 6$ ) a very good and robust correction is achieved (figure 4) [15]. 

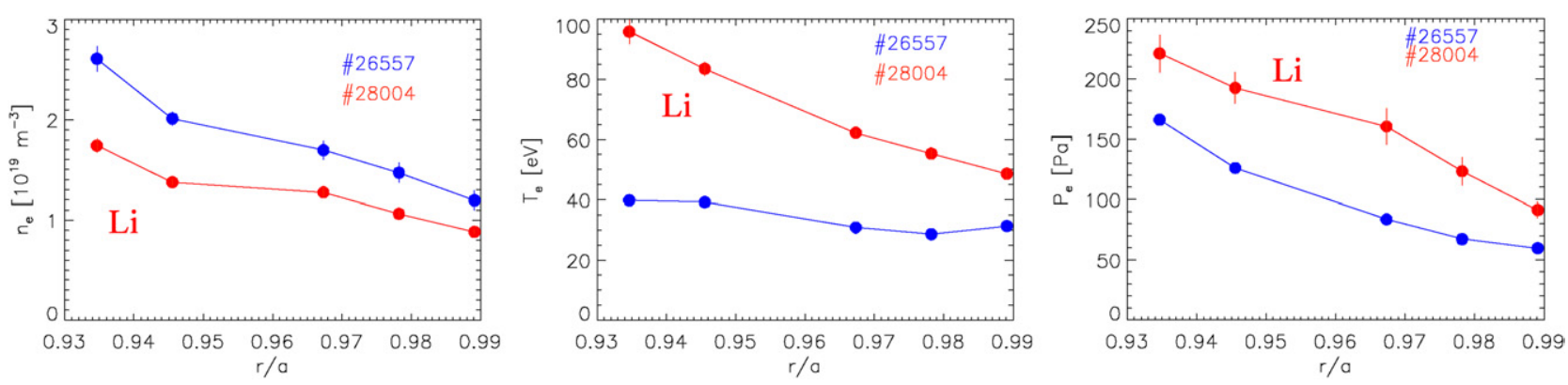

Figure 5. Edge density, temperature and pressure measured by thermal helium beam diagnostic on similar discharges before (\#26557) and after lithization (\#28004).
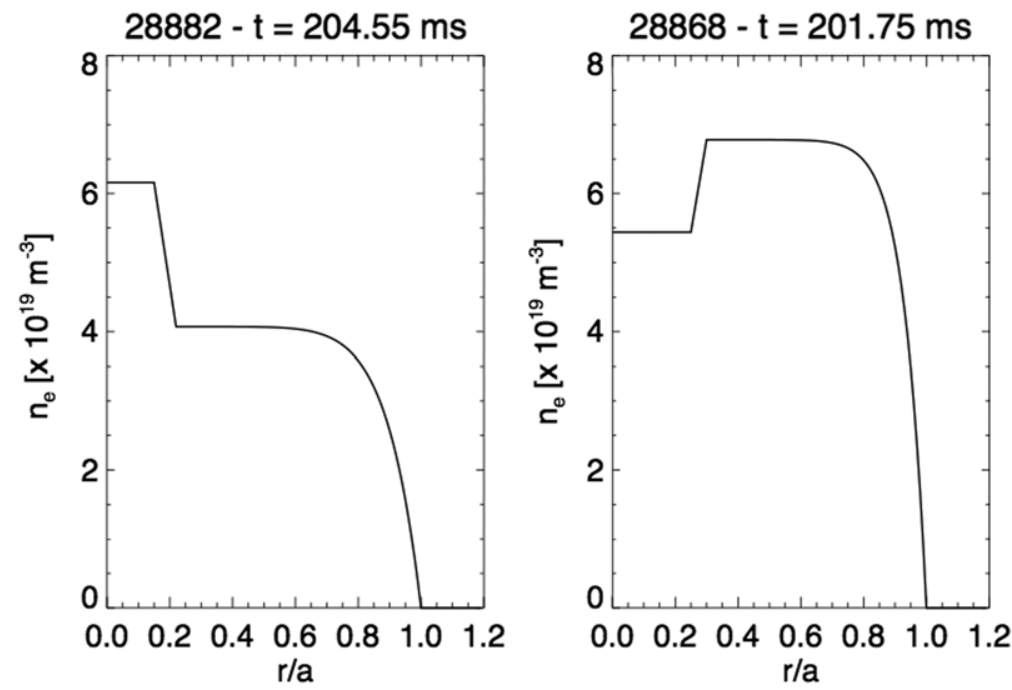

Figure 6. Inverted density profiles with pellet ablation occurring inside (left) or outside (right) the barrier. Inversion is done assuming simplified model profiles.

\subsection{Gain optimization for TM controller}

TMs are always present in RFPs, since they drive the dynamo magnetic self-organization process. Their amplitudes correspond to non-linear saturation and a $k$-spectrum that can be narrow or broad depending on the plasma regime ( $\mathrm{SH}$ or $\mathrm{MH})$. They cannot be fully suppressed and a feedback control system can at best be zeroing the edge amplitudes of their radial components, $b_{r}(a)$. For this purpose optimized gains for each individual active coil need to be used. The optimized proportional-integral-derivative (PID) gains leading to the smallest $b_{r}(a)$ have been calculated by an equilibrium model for a single TM [23] - see section 7, and checked by an experimental scan.

\subsection{Control of the first-wall properties and of density profiles}

In order to reduce graphite first-wall high recycling and improve control of the density profile, in particular at high currents, first-wall lithization has been tested in addition to boronization [13]. Experiments up to now have concentrated on injection of $\mathrm{Li}$ room temperature pellets $(1.5 \mathrm{~mm}$ diameter, $6 \mathrm{~mm}$ length) and only recently a liquid lithium limiter, on loan from FTU [24], has been tested. Li pellet injection in a series of helium discharges provides nearly uniform Li wall deposition. For each campaign a maximum of only $50 \mathrm{Li}$ pellets were injected corresponding to $2 \times 10^{22}$ atoms, $\approx 1 \mathrm{~g}$, for a theoretical coating thickness of about $10 \mathrm{~nm}$. Such a rather low amount of $\mathrm{Li}$, compared with the experience in the literature, is nonetheless effective in maintaining hydrogen wall recycling and impurity influx very low. After lithization edge temperature increases and electron density decreases; overall a higher edge pressure compared with discharges performed before $\mathrm{Li}$ wall conditioning is observed (figure 5). Particle confinement time increases by 20\%. Present experiments are promising and more intensive lithization is expected to produce stronger effects, extending to the core. With low recycling $\mathrm{H}$ pellet injection is an effective way to refuel plasma core, in particular during SH states. Successful injection leads to core peaked density profiles in the presence of ITBs, as shown in figure 6 .

\section{Helical states and ITBs: evidence and understanding}

RFX-mod plasmas at current $\geqslant 1$ MA reproducibly show QSH states with ( $m=1, n=-7)$ helicity. Following the empirical scaling law already discussed in [2], their persistence increases up to $\approx 80-85 \%$ at $I_{\mathrm{p}}=1.8 \mathrm{MA}$. For $I_{\mathrm{p}} \geqslant 1.5 \mathrm{MA}$, QSH turns into SHAx. SHAx states are a necessary condition for eITBs [3,6]. eITB corresponds to a steep electron 


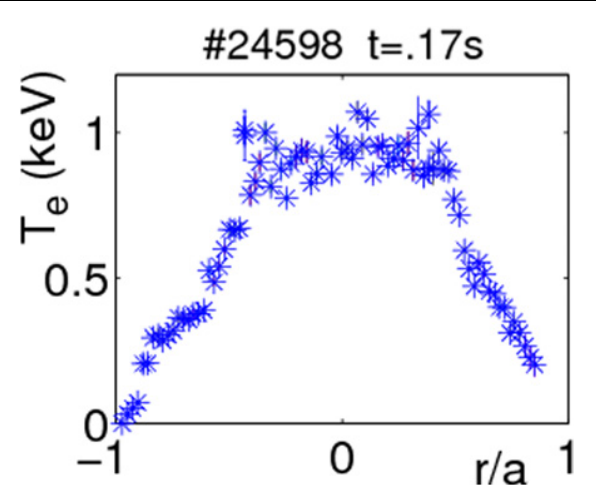

Figure 7. Electron temperature profile with eITB.

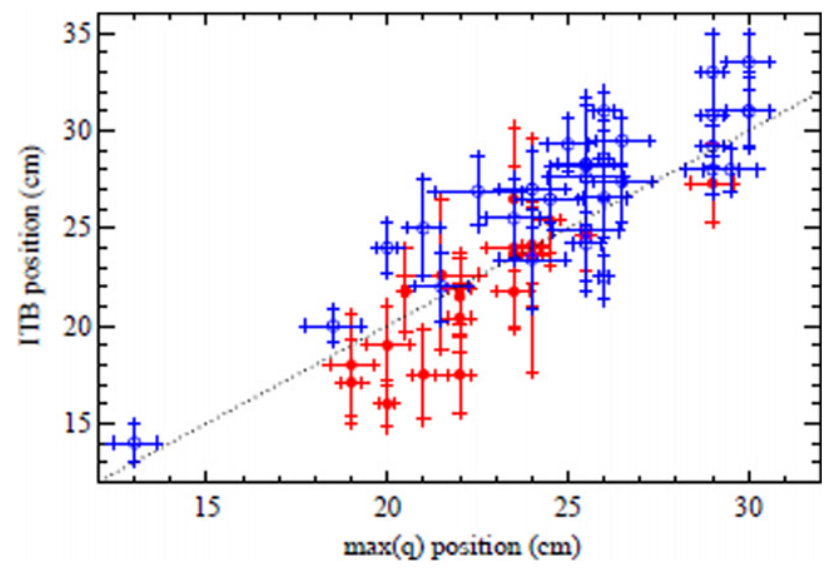

Figure 8. Position of the ITB versus the $q$ maximum location for RFX-mod experimental DAx (full points) and SHAx states (empty points). (DAx: double axis states, see e.g., [12].)

temperature gradient (figure 7), and to a minimum of the thermal conductivity. It also represents a region of reduced particle transport affecting both main gas and impurities.

The location of eITB foot coincides with a point of null shear in the safety factor $q$ profile [11] (see figure 8), which in the case of the RFP corresponds to a $q$ maximum. For a tokamak, where eITBs are also triggered by $q$ reversed shear, a proposed interpretation is based on lower density of rational surfaces and thus on lower probability of mode coupling [25]. In RFX-mod, barriers are also correlated with flow shear. Nonlinear 3D MHD simulations show that the dynamo velocity field features a maximum in the shear profile, which occurs at the location of $q$ maximum [12]. Experimentally, the reconstruction of poloidal flow pattern [6] shows an inversion of the flow internal to the field reversal surface, indicating that flow shear is present, though spatial resolution does not allow discriminating whether higher shear corresponds exactly to maximum $q$. Edge flow shows $(1,7)$ modulation consistent with magnetic topology [14].

In the experiment the ratio between the dominant and secondary modes increases as collisionality gets lower, a condition favouring SHAx (but resistivity may also play a role). eITBs develop at low values of collisionality, with $n / n_{\mathrm{G}} \leqslant 0.25$. This limit is likely due to edge phenomena and in particular to helical PWI (figure 9) [14]. The edge region in QSH is characterized by a chain of $m=0, n=7$ islands, arising as an effect of toroidicity and mode coupling [26].

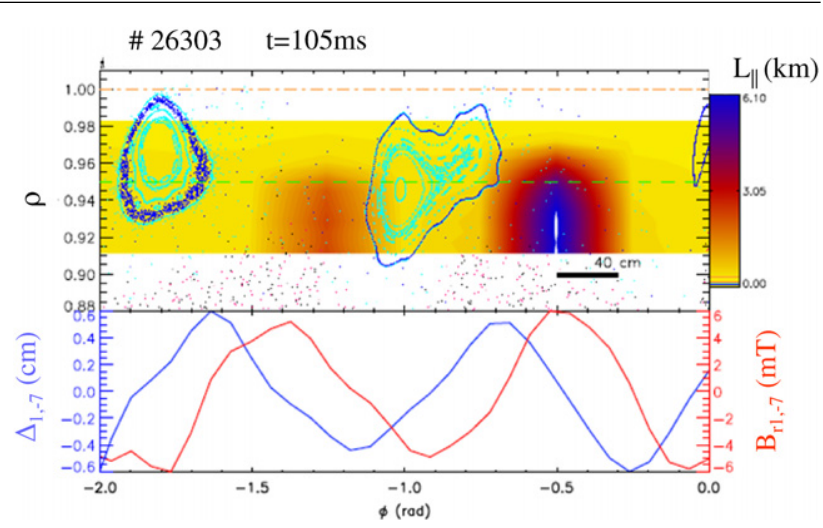

Figure 9. Top: edge Poincaré plot $\left(\theta=0^{\circ}\right)$ of $(0,7)$ island chain and characteristic electron length $L_{\mathrm{II}}$ (colour-coded map); bottom: toroidal behaviour of the shift of dominant mode (blue) and of corresponding radial magnetic field (red).

These islands cause a small helical shift and localized PWI, which determines edge localized density accumulation and plasma cooling. Floating potential has helical modulation, interpreted as a modulation of electron fluxes towards the wall. These localized kinetic perturbations are supposed to facilitate the growth of secondary modes, opposing QSH. Even backtransitions to $\mathrm{MH}$, observed during QSH states, are attributed to reconnection events [27] linked with helical PWI.

Pellet injection is used for refuelling the helical core and creating a peaked density profile since the barrier is also effective for particles: it confines particles inside and prevents penetration from outside. Figure 6 shows two density profiles: on the left a case where ablation occurs in the centre, thus peaking the density; on the right a case where the pellet is ablated outside the barrier and the density profile remains hollow. A similar conclusion is inferred for impurities in experiments with nickel laser blow off and neon puffing [6].

Experimental particle $(\mathrm{H})$ diffusion coefficient $D$ in the barrier is reduced by about one order of magnitude, with $D \leqslant 5 \mathrm{~m}^{2} \mathrm{~s}^{-1}$, and negligible pinch velocity. The field line tracing code ORBIT [38] also indicates that in SHAx the diffusion coefficient at a low collisionality is reduced by about two orders of magnitude with respect to the situation dominated by magnetic chaos, leading to $D \approx 0.5-5 \mathrm{~m}^{2} \mathrm{~s}^{-1}$. This is lower but in the same range as the experimental evaluation. Electron heat diffusivity $\chi_{\mathrm{e}}$ at the barrier decreases to minimum values $\chi_{\mathrm{e}, \min } \approx 5 \mathrm{~m}^{2} \mathrm{~s}^{-1}$. It is worth noting that the value of $\chi_{\mathrm{e}}$ expressed in Bohm units is consistent with similar databases obtained in a tokamak or a stellarator confirming that in the region of eITB the RFP is now approaching the transport quality of the other configurations. Both $\chi_{\mathrm{e}, \mathrm{min}}$ and the electron temperature gradient length $L_{T_{\mathrm{e}}}$ scale inversely with the total amplitude of the secondary $m=1$ modes [6], indicating a strong link between the quality of magnetic topology, i.e. the level of magnetic chaos, and the strength of the barrier.

Preliminary evidence of a limit value for $L_{T_{\mathrm{e}}}$ suggests the presence of other gradient-driven transport mechanisms emerging when magnetic chaos is reduced. Analytical work showed that ITG modes are more stable in RFPs than in tokamaks because of stronger Landau damping [28]. Various numerical tools developed for tokamak turbulence studies have been adapted to RFP: the non-linear electromagnetic 
(flux-tube) gyrokinetic code GS2 [29] and the full-radius fluid (ITG-TEM electrostatic turbulence) code TRB [30]. An integral eigenvalue approach, retaining full finite-Larmorradius effects, has also been used [12]. All agree that ITG modes hardly become linearly unstable under the present experimental conditions. Trapped electron modes (TEM) could arise with density gradients stronger than those now measured. Work to assess the impact of TEM on edge transport is ongoing.

Gyrokinetic calculations show that a strong candidate to limit the temperature gradient is the micro-tearing (MT) instability that is the dominant micro-turbulence mechanism acting on the ion Larmor radius scale. MTs, driven by the electron temperature gradient, may lead to chains of overlapping magnetic islands and to local stochastization of magnetic field lines near mode rational surfaces. In the $T_{\mathrm{e}}$ gradient region MT modes are unstable [12]. Quasi-linear estimate of electron thermal conductivity related to MT falls in the range $\chi \approx 5-20 \mathrm{~m}^{2} \mathrm{~s}^{-1}$, consistent with experimental values. eITBs correspond to better values of the energy confinement time $\left(\approx 5 \mathrm{~ms}\right.$ assuming $\left.T_{\mathrm{e}}=T_{\mathrm{i}}\right)$. Such values of the global confinement time are still not optimal due to the large power still required to sustain the poloidal current flowing in the relatively resistive edge plasma.

Signature of global Alfvén eigenmodes and reversed shear Alfvén eigenmodes is experimentally detected at the plasma edge, though without significant impact on transport [31]. The physics of eITB in RFX-mod has several similarities with that in tokamaks and stellarators. The most striking analogy is with the tokamak, concerning the presence, in both cases, of a null in the $q$ shear.

Analogies also exist with the stellarator: for example, the low collisionality and the presence of a significant flow shear. Both eITB and, as discussed later, the external transport barriers (ETBs - see next section) are found so far in RFXmod in regimes of low collisionality. Whether this analogy is a signature of a similar physics favouring the barrier is an open question. In stellarators, the low collisionality regime is related to the development of a large positive radial electric field $E_{r}$ in the core, and consequently to shared flow (the electron root regime [32]). In RFX-mod, eITBs are strongly coupled to the onset of the SH equilibrium, which is also observed at a low collisionality. Still an open question is whether, in the barrier region of the 3D SHAx topology, transport is described by neoclassical theory and whether an ambipolar electric field shear builds up as in the stellarator e-root regime.

\section{Edge transport barriers}

A new type of very strong electron transport barriers has been observed at the plasma edge $(r / a \approx 0.8)$, where a pedestal up to $1 \mathrm{keV}$ high develops in a few $\mathrm{cm} \mathrm{[6].} \mathrm{Under} \mathrm{these} \mathrm{conditions}$ $T_{\mathrm{e}}$ gradients reach values up to $70-80 \mathrm{keV} \mathrm{m}^{-1}$. An example, more extensively discussed in [6] is shown in figure 10. These edge barriers correspond also to pressure barriers; however, the dominant contribution derives from the temperature profile. ETBs do not necessarily require the presence of a SHAx state and typically appear at low densities. Cases where both eITB and ETB coexist have been found.

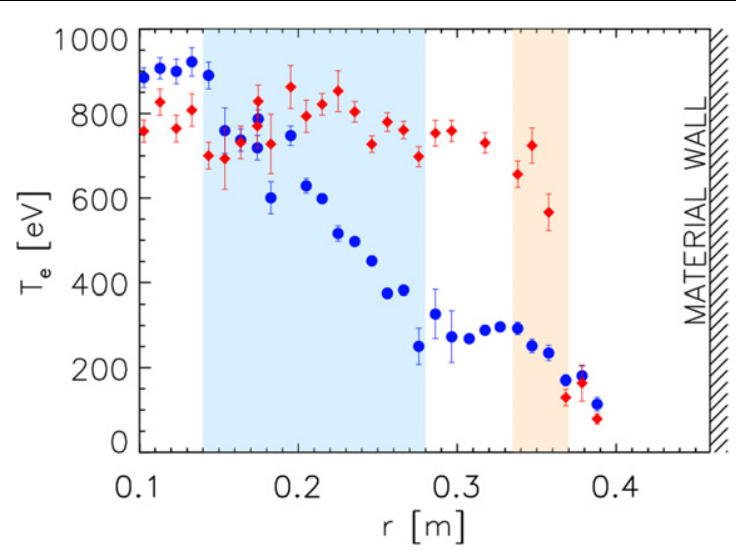

Figure 10. Example of electron temperature profile in the presence of an ETB (red diamonds) compared with a profile in a case with internal barrier (blue full circles) (reproduced with permission from [6]. Copyright 2011 IOP Publishing).

ETBs develop in regimes when secondary modes are relatively low, at low collisionality and prefer regimes with shallow edge toroidal magnetic field reversal. Magnetic chaos reduction appears to be a common feature of both internal and external barriers. ETBs develop in fact in a region characterized by ordered magnetic surfaces, as shown by the field line tracing reconstructions made with the FliT code [33]. The existence of a significant edge transport barrier expands the possibilities of operating improved confinement RFP regimes.

\section{Building a 3D knowledge}

The discovery of SHAx states gives a unique opportunity to investigate the physics of $3 \mathrm{D}$ fields in magnetized fusion plasmas and to broaden the knowledge basis in this area. There is, in fact, a growing interest both in the stellarator and in the tokamak community on the effects of 3D shaping of the magnetic field. The study of 3D RFX-mod features benefit from the widely known codes originally developed for the stellarator and adapted to the RFP in a growing collaborative effort.

Helical magnetic equilibrium is described analytically and numerically via both a perturbative analysis in toroidal geometry and a full 3D approach.

The analytical calculation [12] of ohmic RFP SH states is performed in the frame of resistive MHD in cylindrical geometry, using perturbation theory for a paramagnetic pinch with low edge conductivity and axial magnetic field. A necessary criterion for toroidal field reversal at the edge is derived. The criterion involves the radial profile of the logarithmic derivative of the Newcomb eigenfunction of the pinch. It is suggestive that a finite edge radial magnetic field might be favourable for field reversal.

In the numerical perturbative approach, implemented in the SHEq code [11], the zero-order toroidal force balance is solved together with the Newcomb equation, which provides helical field components [11]. Full 3D calculation is performed with VMEC [34], adapted to RFP using poloidal flux as a magnetic surface label [11]. In fixed boundary runs the magnetic surfaces match the prediction of both field line tracing codes and the analytical approach. While the magnetic field 


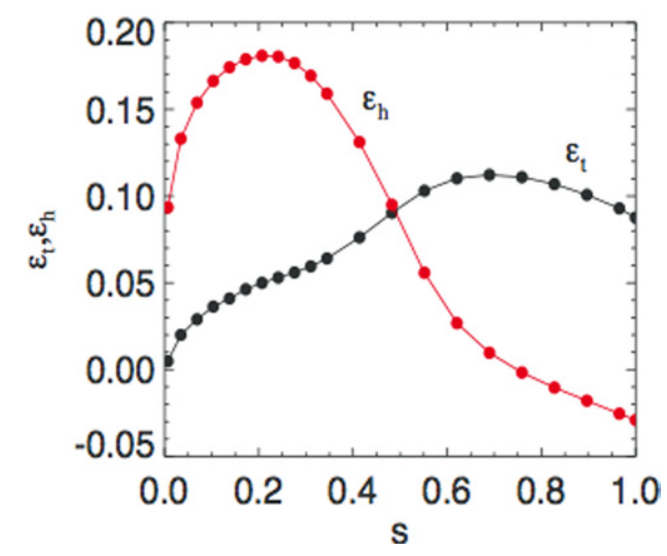

Figure 11. An indicator of the deviation from axis-symmetry of the RFX-mod equilibrium magnetic field: the radial profiles of the helical $\varepsilon_{\mathrm{h}}$ and toroidal $\varepsilon_{\mathrm{t}}$ ripples for RFX-mod in a typical SHAx state ( $s$ is the normalized poloidal flux computed in the helical equilibrium, $\varepsilon_{\mathrm{h}}$ and $\varepsilon_{\mathrm{t}}$ defined as in the stellarator [11]).

topology is helical, $|\boldsymbol{B}|$ keeps an approximate axisymmetric shape. The RFP free-boundary version of VMEC, under development, will allow modelling an externally imposed magnetic field. Investigation on the use of externally applied rotational transform is underway [35]. SHEq 3D equilibria are used as input to the ASTRA [36] code to interpret experimental transport measurements. Preliminary analysis of ideal stability with the Terpsichore code [37] indicates that symmetry breaking modes $(1,8)$ and $(2,15)$ are more stable with core-reversed shear than with null shear.

3D fields also play an important role in determining transport. In SHAx, neoclassical effects, together with microturbulence, might have a significant role in ruling transport in the helical core and across internal barriers. Two main approaches for numerical study of transport in helical states are used. The first is based on the ORBIT code [38]: volumeaveraged particle transport coefficients across helical surfaces are estimated numerically by a mono-energetic test particle approach [39]. This approach takes into account both the drifts of particle trajectories and the effect of the residual magnetic chaos. Work is in progress to implement in ORBIT also an electric field perpendicular to helical surfaces. A complementary approach is based on DKES [40], in order to estimate the full matrix of local neoclassical transport coefficients. This is based on the assumption that transport is described by a local approach.

As done in stellarators deviations from axis-symmetry are described by means of the radial functions $\varepsilon_{\mathrm{h}}(\mathrm{s})$ and $\varepsilon_{\mathrm{t}}(\mathrm{s})$, the helical and the toroidal ripple, respectively [11]. As shown in figure $11, \varepsilon_{\mathrm{h}}$ is dominant in the central region $\left(\varepsilon_{\mathrm{h}} \approx 2-3 \varepsilon_{\mathrm{t}}\right)$ and $\approx 0$ at the edge $\left(\varepsilon_{\mathrm{h}} \approx 0.1-0.2 \varepsilon_{\mathrm{t}}\right)$. Thus, while the core is strongly helically deformed, the outer region almost preserves typical properties of a quasiaxisymmetric configuration. Neoclassical effects, and in particular super-bananas, which affect unoptimized stellarators at low collisionality, might not be a significant issue. ORBIT shows that when trapped particles drift out of the helical core with high $\varepsilon_{\mathrm{h}}$, they reach a region $(r / a \approx 0.6)$ where $\varepsilon_{\mathrm{h}}$ decreases. They become almost passing without being lost, at least at a low collisionality. ORBIT Monte Carlo simulations confirm that ion diffusion coefficient $\left(D_{\mathrm{i}}\right)$-volume-averaged over the helical domain-plotted versus collisionality $v$ does not show the $1 / v$ regime typical of unoptimized stellarators (figure 12).

The addition of self-consistent heat transport dynamics to 3D non-linear MHD codes is expected to provide significant improvements in modelling of RFP self-organization [12]. To this end the PIXIE3D initial value code has been implemented for RFP. Recently, careful benchmarking against the SpeCyl code has successfully completed the mandatory step of numerical verification [12].

\section{Active control of MHD stability}

Research on feedback control of MHD stability is providing key results [15]. Real-time control experiments have been performed operating RFX-mod both as RFP and as tokamak, to address RWMs, TMs and error field control.

Improvements are obtained by including in control models toroidal geometry and deviations from uniformity of passive structures. A first technique is based on a stationary decoupling matrix, which is effective in producing pure harmonics at the sensor radius. The dynamical pseudo-decoupler is a more elaborated tool, which takes into account the frequency dependence of the toroidal (poloidal) coupling. As previously shown (section 3), its use substantially decreases time varying toroidally localized field errors in the start-up phase.

3D features of the magnetic boundary, such as gaps in the resistive wall and portholes, influence RWM growth rates and spatial structure. Many poloidal harmonics contribute to the mode structure, even in the case of circular plasmas like that of RFX-mod. Mode non-rigidity has been studied. The 192 active coils set can be on purpose downgraded and reconfigured to test the role of coil geometry and number in stabilization. This is done in collaboration with JT-60SA to help the design of its set of active coils [41]; the most unstable RWM can still be controlled by reducing the active coil number up to $1 / 24$ (surface covered by coils down to $4.2 \%$ ) after tuning of the gains.

RFX-mod is an excellent tool to benchmark against experimental data complex numerical codes used to predict plasma stability and to simulate the behaviour of real-time controllers. Accurate modelling of MHD stability and control is indeed a key request for ITER. This requires understanding the effects on stability of plasma rotation and of the complex 3 D conducting structures surrounding the plasma. Since RWM stability in RFP is not affected by dissipation and flow [15], RWM modelling can focus on 3D effects removing other sources of uncertainties. A 3D model of RFX-mod has been implemented in the code CarMa and benchmarked against RFX-mod RWM experimental data [42]. A cylindrical MHD model including plasma pressure and longitudinal flow has also been benchmarked against RFX-mod data [43].

A new integrated simulator for closed-loop control experiments has been developed and benchmarked [44]. The tool couples self-consistently a full $3 \mathrm{D}$ description of the machine boundary (Cariddi code), a 2D toroidal model of stability (MARS) and a dynamic model of the control system cast in the state variable representation. Using actual PID gains and plasma equilibrium parameters such a 'flight 

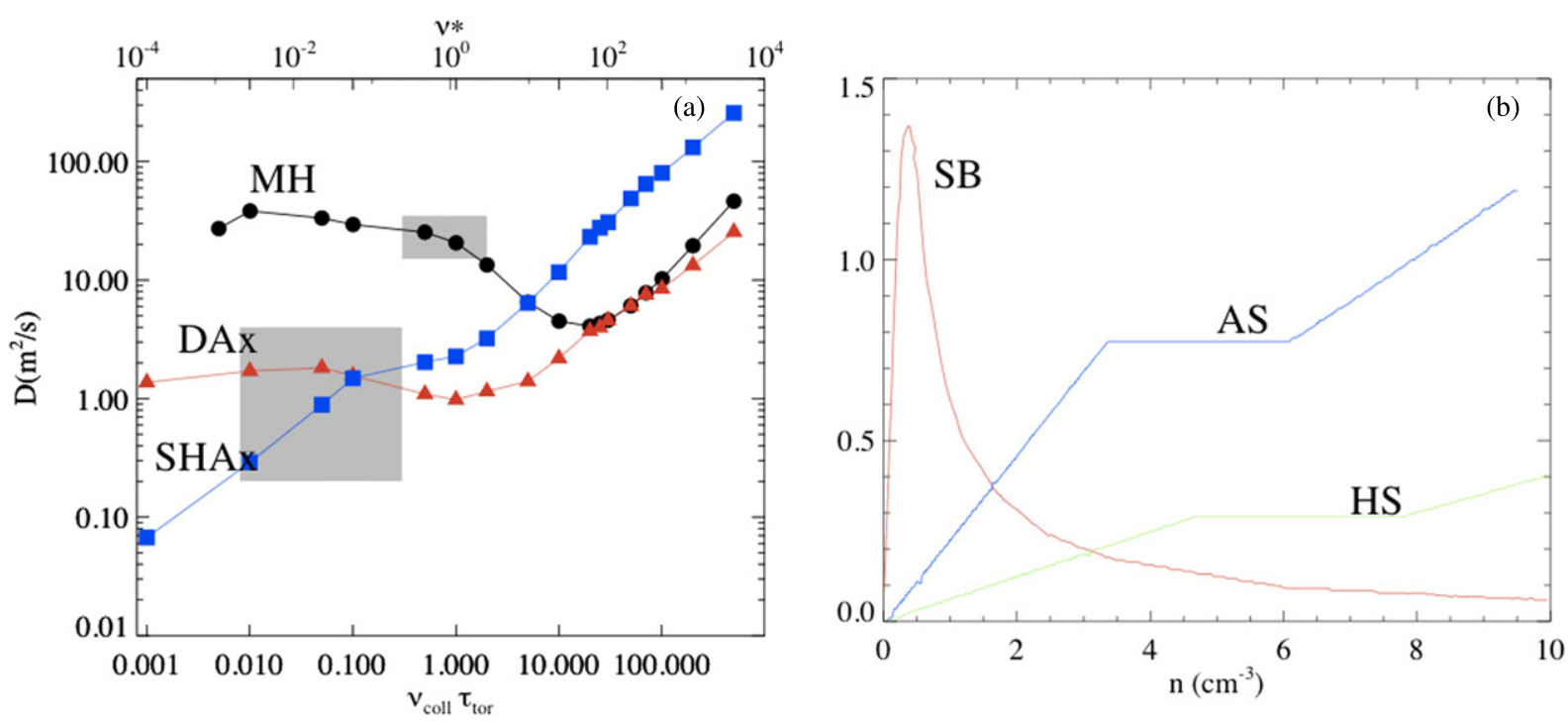

Figure 12. Left: diffusion coefficient $D$ of $\mathrm{H}+$ ions as a function of $v_{\text {coll }} \tau_{\text {tor }}$ (black $=\mathrm{MH}$, red $=\mathrm{DAx}$, blue $=\mathrm{SHAx}$ ). Right: schematic view of the neoclassical transport regimes: AS = axisymmetric system, HS = helically symmetric, $\mathrm{SB}=$ superbanana (adapted from [45]). DAx states are those where a large helical structure is present together with the original axis-symmetric axis (island configuration).

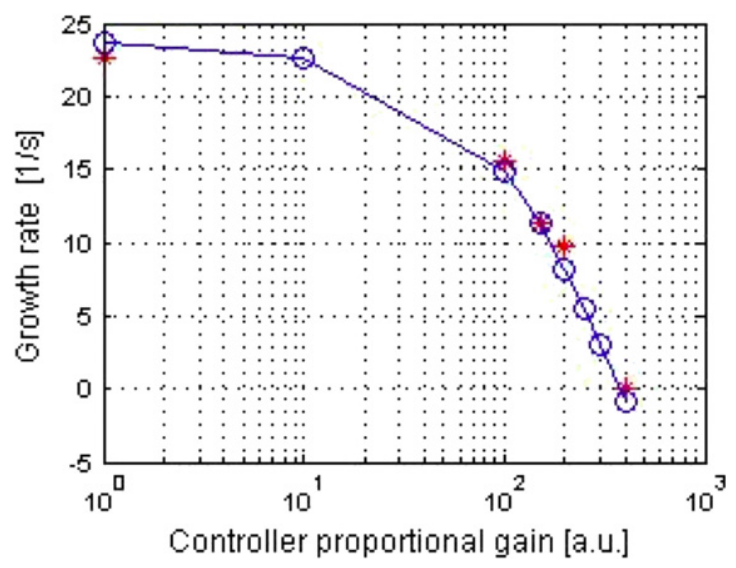

Figure 13. Numerical growth rates computed as a function of the controller gain (continuous curve-open dots) compared with experimental ones measured with high accuracy under the same controller conditions, i.e. using the same gains (asterisks) [44].

simulator' successfully reproduces experimental closed-loop RWM growth rates, as shown in figure 13.

Control of resonant TMs is more challenging. Several TMs are linearly unstable in the RFP, and non-linearly reach saturated amplitude. Their dynamics under feedback control conditions is highly non-linear: the increase in proportional gain reduces the edge radial field to a minimum; any further increase induces mode rotation. Since the last FEC, an intense activity to optimize feedback laws for resonant modes has been undertaken. Basic effects of the clean mode control (CMC) are explained in [23] using an equilibrium model for a single TM. The model assumes the multiple shell structures of RFX-mod and is based on the balance between the electromagnetic torque-produced by the conductive structures and the feedback coils surrounding the plasmaand the viscous torque due to the fluid motion. Recently, the model has been upgraded and now a more accurate diffusion equation, which takes into account the shell thickness, is used to describe the shell penetration [23]. A more complete multimode dynamic model, implemented in the RFXlocking code [23], is also developed. This model evolves the mode frequencies and edge amplitudes of several TMs at the same time, taking into account not only the interaction with the external structures but also the non-linear interaction between themselves. The model has driven the experimental PID gain optimization previously discussed.

\section{Tokamak operation in RFX-mod}

Thanks to its high flexibility, RFX-mod can be run as a tokamak, with $120 \mathrm{kA}$ plasma current and discharge duration up to $1.2 \mathrm{~s}$. Plasma current is basically limited by the available toroidal field, which is not very large since the coils are designed with the RFP target.

Tokamak plasmas were used to study edge turbulence with the same tools used in the RFP. More recently, a project has started to use RFX-mod tokamak plasmas as a test-bed for real-time MHD active stability control. Even if the RFX-mod tokamak has a low current, it shares several MHD instabilities with a larger device, which need to be controlled, and has the unique feature of a high-performance system of active coils. In this configuration a current-driven $(2,1)$ RWM is observed in ramped current plasmas as $q_{\text {edge }} \approx 2$ ( $q_{\text {edge }}$ is reconstructed from external measurements). The experiment follows an idea proposed for DIII-D [45-48] and HBT-EP [49] to excite current-drive external kink modes and then implemented in RFX-mod. Feedback control allows full stabilization of this mode, and for the first time a feedback stabilized $q_{\text {edge }} \approx 2$ tokamak plasma is run without disruptions (figure 14).

\section{Conclusions}

Since December 2004, when experiments started in the RFX-mod device, the RFX fusion science program has 


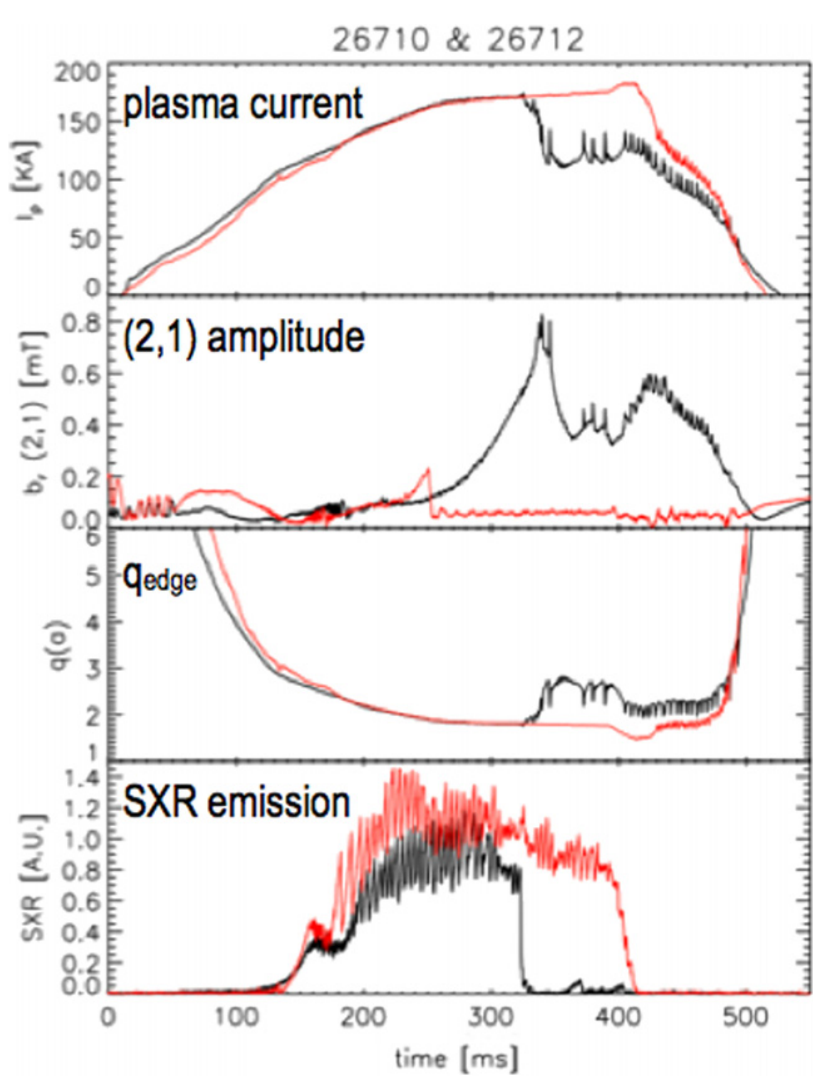

Figure 14. Example of RFX-mod ramped current tokamak operation. Black curves: without feedback; growth of $(2,1)$ is evident. Red curves: with feedback; the mode is kept at a very low value.

successfully reached a number of key milestones and new and transformational results have been obtained.

A number of issues remain to be solved in the RFXmod route for a full assessment of the RFP fusion potential. First-wall behaviour-in particular in terms of recyclingand in general control of density profiles in helical states need to be optimized. This will help both in enhancing plasma thermal content and in avoiding cool and resistive edge plasmas, a feature that has a significant cost in terms of input power. Further optimization of the quality of the magnetic boundary will also be helpful in reducing edge magnetic turbulence and magnetic driven transport, and in making the helical states more robust. Improvements on these two issues may lead to stronger and more reproducible edge pressure pedestal, an important requirement for a global improvement of confinement. More long-range activities on current drive (experiments on oscillating field current drive started in RFX-mod), on tools for edge lost particle disposal and on optimization of the feedback system are going on. The device flexibility helps in testing proof of principle solutions.

Confidence on the path for the solution of these issues is given by the rich set of results obtained since 2004, part of which is reported in this paper. The discovery of self-organized helical states with electron internal transport barriers, and the achievement of core electron temperature $\geqslant 1.5 \mathrm{keV}$, is a demonstration that RFP is not necessarily plagued by high magnetic transport. Active control of MHD stability proves that a thick shell is not necessary, and provides a very flexible tool to explore a number of ITER-relevant questions. Unique in the fusion arena, RFX-mod can operate with active control both in tokamak and RFP configurations. A growing subject like that of three-dimensional fusion physics has RFX-mod as an active player, with strong integration with stellarator and tokamak communities. RFX-mod has also supported the community with new contributions to key topics like the density limit of turbulence driven transport. The completion of on-going diagnostic projects and the installation of a $1 \mathrm{MW}$, $25 \mathrm{kV} \mathrm{H}$ beam, on loan from AIST Tsukuba, will enhance the portfolio of tools for exploring RFP physics also on new topics, like fast ions.

RFX-mod is in an excellent position to take up the challenge of setting a milestone in the route for the assessment of RFP potential as an ohmic, low magnetic field, high engineering beta fusion reactor, and to be an effective player in the fields of real-time feedback control of MHD stability and of three-dimensional fusion physics.

\section{Acknowledgment}

The authors wish to thank the whole technical and administrative staff of Consorzio RFX for the continuous and generous support.

\section{References}

[1] Sonato P. et al 2005 Fusion Eng. Des. 7497

[2] Martin P. et al 2009 Nucl. Fusion 49104019

[3] Lorenzini R. et al 2009 Nature Phys. 5570

[4] Fantini F. et al $2010 \mathrm{New}$ cooling system with dielectric fluid for magnetizing winding in RFX-mod experiment Presented at the 26th SOFT Conf. (Porto, 2010) http://portal. ipfn.ist.utl.pt/soft2010_abstractbook/pdf/P3-031.pdf

[5] Zanotto L. et al 2010 Optimization of the RFX-mod experiment for 2 MA operation Proc. 37th EPS Conf. on Plasma Physics (Dublin, Ireland, 2010) paper P2.193, http://ocs.ciemat.es/EPS2010PAP/pdf/P2.193.pdf

[6] Puiatti M.E. et al 2011 Internal and edge electron transport barriers in the RFX-mod reversed field pinch Nucl. Fusion 51073038

[7] Kishimoto Y. et al 2000 Nucl. Fusion 40667

[8] Hogeweij G.M.D. et al 2002 Plasma Phys. Control. Fusion 441155

[9] Coda S. et al 2007 Nucl. Fusion 47714

[10] Piovesan P. et al 2011 Role of external 3D magnetic fields and flow in helical RFX-mod equilibria Plasma Phys. Control. Fusion submitted

[11] Marrelli L. et al 2010 Proc. 23rd Int. Conf. on Fusion Energy 2010 (Daejeon, Korea, 2010) (Vienna: IAEA) CD-ROM file EXS/P5-10 and http://www-naweb.iaea.org/napc/physics/ FEC/FEC2010/html/index.htm

[12] Cappello S. et al 2011 Equilibrium and transport for quasi helical reversed field pinches Nucl. Fusion submitted

[13] Dal Bello S. et al 2010 Proc. 23rd Int. Conf. on Fusion Energy 2010 (Daejeon, Korea, 2010) (Vienna: IAEA) CD-ROM file EXD/P3-06 and http://www-naweb.iaea.org/nape/ physics/FEC/FEC2010/html/index.htm

[14] Scarin P. et al 2011 Magnetic structures and pressure profiles in the plasma boundary of RFX-mod: high current and density limit in helical regimes Nucl. Fusion $\mathbf{5 1} 073002$

[15] Bolzonella T. et al 2010 Proc. 23rd Int. Conf. on Fusion Energy 2010 (Daejeon, Korea, 2010) (Vienna: IAEA) CD-ROM file EXS/P5-01 and http://wwwnaweb.iaea.org/napc/ physics/FEC/FEC2010/html/index.htm

[16] Martin P. 1999 Plasma Phys. Control. Fusion 41 A247-55 
[17] Escande D.F. et al 2000 Phys. Rev. Lett. 85 1662-5

[18] Brunsell P. et al 2004 Phys. Rev. Lett. 93225001

[19] Martin P. 2011 Fusion Sci. Technol. 59 602-16

[20] Hender T. et al 2007 Progress in the ITER Physics Basis: chapter 3. MHD stability, operational limits and disruptions Nucl. Fusion 47 S128-S202

[21] Stoneking M.R. et al 1998 Phys. Plasmas 51004

[22] Soppelsa A. et al 2008 Fusion Eng. Des. 83 224-7

[23] Zanca P. 2009 Plasma Phys. Control. Fusion 51015006

[24] Apicella M.L. et al 2007 J. Nucl. Mater. 363-365 1346

[25] Garbet X. et al 2001 Phys. Plasmas 82793

[26] Spizzo G. et al 2006 Phys. Rev. Lett. 96025001

[27] Zuin M. et al 2009 Plasma Phys. Control. Fusion 51035012

[28] Guo S.C. 2008 Phys. Plasmas 15122510

[29] Predebon I. et al 2010 Phys. Plasmas 17012304

[30] Sattin F. et al 2010 Plasma Phys. Control. Fusion 52105002

[31] Spagnolo S. et al 2010 Alfvén eigenmodes in the RFX-mod RFP plasma Proc. 37th EPS Conf. on Plasma Physics (Dublin, Ireland, 2010) paper P4.162, http://ocs.ciemat.es/EPS2010PAP/ pdf/P4.162.pdf

[32] Takeiri Y. et al 2003 Phys. Plasmas 101788

[33] Innocente P. et al 2007 Nucl. Fusion 471092

[34] Hirshman S.P. and Whitson J.C. 1983 Phys. Fluids 263554

[35] Boozer A.B. and Pomphrey N. 2009 Phys. Plasmas 16022507
[36] Pereverzev G et al 2002 Report IPP 5/98, Garching (February 2002)

[37] Cooper W.A. et al 2006 Fusion Sci. Technol. 50245

[38] White R.B. and Chance M.S. 1984 Phys. Fluids 272455

[39] Gobbin M. et al 2009 Plasma Phys. Control. Fusion 51065010

[40] Hirsman S.P. et al 1986 Phys. Fluids 292951

[41] Takechi M. et al 2010 Mode rigidity study of RWM on RFX with reduced RWM control coils for JT-60SA RWM stabilization Proc. 37th EPS Conf. on Plasma Physics (Dublin, Ireland, 2010) paper P-2.192, http://ocs.ciemat.es/EPS2010PAP/ pdf/P2.192.pdf

[42] Villone F. et al 2008 Phys. Rev. Lett. 100255005

[43] Wang Z.R. et al 2010 Phys. Plasmas 17052501

[44] Marchiori G. et al 2010 RWM studies on RFX-mod with dynamical controllers: modelling and experimental results Proc. 37th EPS Conf. on Plasma Physics (Dublin, Ireland, 2010) paper P2.183, http://ocs.ciemat.es/EPS2010PAP/pdf/P2.183.pdf

[45] Mynick H.E. 2006 Phys. Plasmas 13058102

[46] In Y. et al 2010 Nucl. Fusion 50042001

[47] In Y. et al 2010 Plasma Phys. Control. Fusion 52104004

[48] Okabayashi M. et al 2009 Nucl. Fusion 49125003

[49] Cates C. et al 2000 Phys. Plasmas 73133 